\title{
Flight factors influence on human lymphocyte radioadaptive response and gamma-interferon production
}

\author{
Elena Arkhipova, Irina Alchinova, Mikhail Karganov \\ Lab of Physicochemical and Ecological Pathophysiology, Institute of General Pathology and Pathophysiology, Moscow, Russia
}

\section{Email address:}

hippova@gmail.com (E. Arkhipova)

\section{To cite this article:}

Elena Arkhipova, Irina Alchinova, Mikhail Karganov. Flight Factors Influence on Human Lymphocyte Radioadaptive Response and Gamma-Interferon Production. American Journal of Life Sciences. Special Issue: Space Flight Factors: From Cell to Body. Vol. 3, No. 1-2, 2015, pp. 43-47. doi: 10.11648/j.ajls.s.2015030102.17

\begin{abstract}
Interferon- $\gamma$ (IFN- $\gamma$ ) is a pleiotropic cytokine with antiproliferative and immunomodulatory activities that are crucial for the regulation of immune responses. We examined a group of military pilots. The examinees were divided into 3 subgroups: ground personnel (9 persons, control group), 17 pilots with $<1000 \mathrm{~h}$ flight time, and 12 pilots with $>1000 \mathrm{~h}$ flight time. No differences in IFN- $\alpha$ serum content after induction by NDV virus were detected. The quality of reparation is in many respects genetically determined; therefore, we used peripheral blood lymphocytes from pilots for in vitro detection of a radioadaptive response (RAR), which was evaluated by the number of chromosome aberrations. The adaptive response was observed in 7 individuals of the control group (78\%), in 10 pilots who had $<1000$ flight hours (59\%), and in 4 pilots having >1000 flight hours (33\%). The examined individuals were divided into 2 groups depending on the presence of RAR, and IFN- $\gamma$ production after radiation was measured. It was shown that at doses 0.05 Gy or 0.5 Gy no differences between groups were detected. Exposure with these doses sequentially in $48 \mathrm{~h}$ interval resulted to differently directed changes: lymphocytes of individuals with RAR produced more IFN- $\gamma$ than before while cells of persons without RAR made it less. The quality of adaptive mechanists evaluated by RAR may be useful for estimation of individual sensitivity to radiation during radiotherapy in oncology and in prediction of professional risk.
\end{abstract}

Keywords: Radioadaptive Response, Flight Factors, Interferon- $\gamma$, Individual Sensitivity

\section{Introduction}

Air crew is exposed to various potentially dangerous factors during flights. The main problem in evaluation of biological risk of anthropogenic and other influences in modern aviation is the necessity to analyze the effect of doses not surpassing or slightly surpassing the threshold doses. In this range the response to the studied factors is maximally individual. Biological consequences of these influences can be revealed only in multilevel studies.

Complex examination of passenger aircraft crew performed by Cavallo et al. has demonstrated that flight factors induced changes in some cytogenetic parameters [1]. In particular, the study revealed higher frequency of chromatid exchanges (4.6 vs. 3.8) and increased (by 1.3 times) frequency of chromosome aberrations in crew members in comparison with the control. Analysis of DNA damage in buccal epithelial cells by comet assay has shown that this parameter in the examined group surpassed the control value. Oxidative DNA damages were found in $9.7 \%$ buccal epithelial cells and in $14.6 \%$ lymphocytes in the examined group and were absent in the control.

Increased radiation level is related to solar activity and depends on flight altitude. Under conditions of maximum solar activity, equivalent doses at altitudes of $6,000,9,000$, and $12,000 \mathrm{~m}$ are $9.9 \times 10^{-7}, 3.35 \times 10^{-6}$, and $7.29 \times 10^{-6} \mathrm{~Sv} / \mathrm{h}$, respectively. The integral doses for total flight time of $1000 \mathrm{~h}$ are $9.9 \times 10^{-4}, 3.35 \times 10^{-3}, 7.29 \times 10^{-3} \mathrm{~Sv}$, respectively [2]. Since these doses correspond to low dose range, the individual reaction of the organism to radiation and other flight factors cannot be predicted. High radiation level in combination with other stress factors can induce damage to cell structures, changes in their functioning, which, in turn, can lead to activation of defense systems. Long-term chronic exposure to extreme factors leads to exhaustion of the adaptive resources and under these conditions hypersensitivity of the organism can develop even after subthreshold exposures. The use of irradiation as a tool for evaluation of individual reactions enables strict control of the exposure. Moreover, irradiation 
is a nonspecific factor triggering nonspecific cell defense mechanisms, which makes possible to predict the response to a wide spectrum of influences.

Biological dosimetry is based on the fact that the factor induces a certain power-, exposure-, and dose-depending response in the majority of the sample; however, some individuals evidently demonstrate extreme responses. Population studies aimed at the evaluation of spontaneous level of chromosome aberrations have demonstrated that there always are some individuals in large samples that differ (without appreciable reasons) by enhanced or reduced level of damages [3].

\section{Materials and Methods}

The following methods were used in the study: evaluation of the incidence of radioadaptive response (RAR) in peripheral blood lymphocytes for evaluation of adaptive capacities of the organism to flight factors in pilots.

Radioadaptive response is a biological phenomenon in which resistance to a challenging dose of radiation is established by one or several very small preceding doses.

We examined a group of military pilots. No differences in IFN- $\alpha$ serum content after induction by NDV virus were detected. The quality of reparation is in many respects genetically determined; therefore, we used peripheral blood lymphocytes from pilots for in vitro detection of a radioadaptive response (RAR), which was evaluated by the number of chromosome aberrations.

We examined a group of military pilots. The examinees were divided into 3 subgroups: ground personnel (9 persons, control group), 17 pilots with $<1000 \mathrm{~h}$ flight time, and 12 pilots with $>1000 \mathrm{~h}$ flight time.

This group includes individuals subjected to strict and regular medical examinations, but at the same time exposed to a complex of flight factors, such as vibration, acceleration forces, emotional stress, and ionizing radiation [1].

Adverse effects of high concentrations of mutagens on cells in some cases can be attenuated by preliminary exposure to the same mutagen in a low dose. In proliferationstimulated human peripheral blood lymphocytes in vitro exposed to acute low-LET irradiation in a dose of a few Gy, the real yield of DNA damages was below the expected level if the cells were preliminary irradiated in a dose of tens $\mathrm{mGy}$. This effect of low doses was called as induction of the adaptive response (AR). In the literature, the first low (preliminary) dose is called conditioning or adaptive dose (AD) and the second main dose is called testing or damaging dose (DD) [4]. AR is associated with cell capacity to repair DNA damages. It is closely related to cell metabolism and changes in the cell that is considered as a response to genotoxic stress (stress response).

The study was performed on PHA-stimulated human peripheral blood lymphocytes. The following reagents were used for cell culturing: cytotoxicity- and mycoplasma-tested sterile fetal calf serum (Perbio-HyClone, USA; $1.6 \mathrm{ml}$ per flask), RPMI-1640 nutrient medium with $25 \mathrm{mM}$ HEPES and sodium bicarbonate $(6.2 \mathrm{ml}$ per flask), and PHA (phytohemagglutinin-P from Phaseolus vulgarios, Sigma, USA; $0.15 \mathrm{ml}$ per flask). Whole heparinized blood was transferred to glass flasks for cell culturing ( $1 \mathrm{ml}$ per flask). For evaluation of individual sensitivity to radiation, an irradiation scheme for detection of the adaptive response was applied.

Irradiation was carried out on a $\gamma$-device using a beam power of $25 \mathrm{rad} / \mathrm{min}(0.25 \mathrm{~Gy} / \mathrm{min})$ at a distance of $65 \mathrm{~cm}$.

Adaptation dose of 0.05 Gy was applied during seeding at G0 stage of the mitotic cycle. Two flasks were irradiated simultaneously: one flask was used for evaluation of the effect of the adaptation dose and another was exposed to the challenging dose after $48 \mathrm{~h}$. In parallel, an intact flask with the blood was exposed to the challenging dose of $0.5 \mathrm{~Gy}$. Control flask with the blood was not irradiated. The cells were incubated in a thermostat at $37^{\circ} \mathrm{C}$ after seeding until fixation (Fig.1).

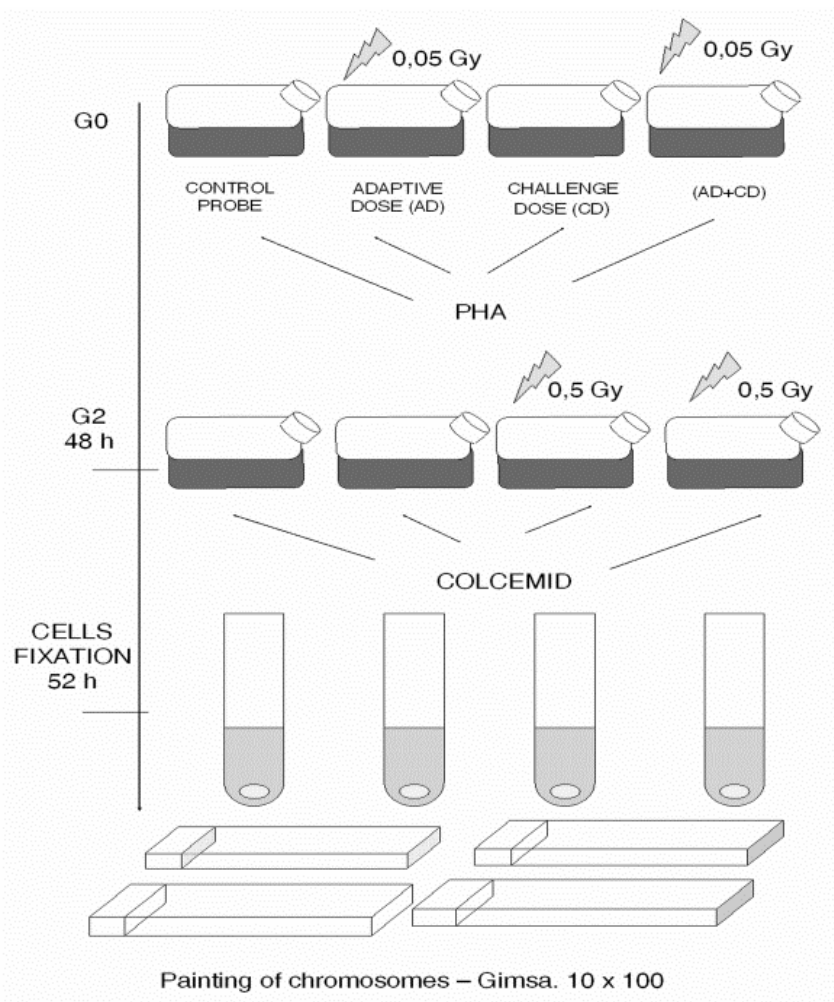

Figure 1. Scheme of the experiments

Fixation. The cells were fixed after $52 \mathrm{~h}$. Two hours before fixation, colcemid was added to each flask ( $0.17 \mathrm{ml}$ per flask) for arresting cell division during metaphase and obtaining metaphase plates. After incubation the cells were centrifuged at $1000 \mathrm{rpm}$ for $15 \mathrm{~min}$. The supernatant was removed, 0.5 $\mathrm{ml}$ pellet was suspended in $10 \mathrm{ml}$ hypotonic $\mathrm{KCl}$ solution, thoroughly mixed, and incubated in a thermostat at $37^{\circ} \mathrm{C}$ for $15 \mathrm{~min}$. The cell suspension was centrifuged again at 1000 rpm and the supernatant was removed. The pellet was stirred on a magnetic stirrer and a fixative (3:1 methanol-glacial acetic acid mixture) was added dropwise. Several portions of fixative are usually changed. 
Preparation processing. The preparations were made by dropping $0.5 \mathrm{ml}$ cell suspension in fixative onto wet cold glasses followed by drying on air for several hours. The preparations for routine analysis were stained after Giemsa and analyzed under a microscope in transmitted light under oil immersion at $\mathrm{x} 1000$. The following chromosome aberrations were counted: chromatid fragments, isochromatid fragments, and symmetrical and asymmetrical chromatid exchanges (Fig. 1) The presence of the adaptive response was detected by the ratio of chromosome and chromatid aberrations at various irradiation doses $(\mathrm{RAR}=(\mathrm{CON}+\mathrm{CD}) /(\mathrm{AD}+\mathrm{CD})) . \quad \mathrm{RAR} \geq 1.5 \quad$ indicates the presence of the adaptive response [4].

In each donor, 100 metaphase plates were analyzed at each term of the experiment (Fig. 2).

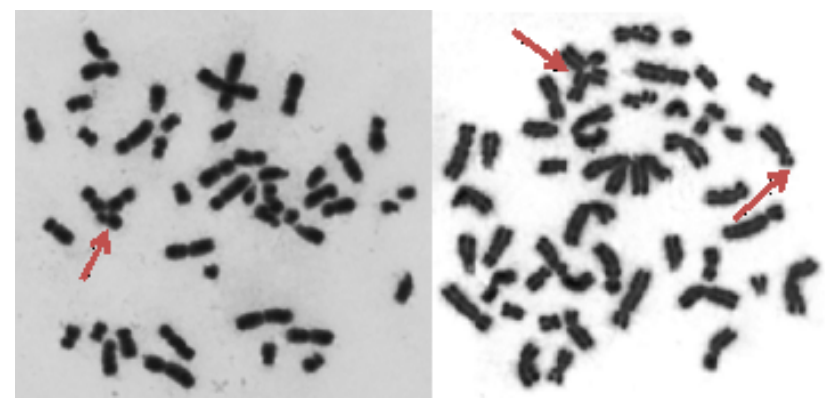

Figure 2. Examples of chromosome aberrations

Experimental protocol: according to Mortazavi, G0 $\rightarrow \mathrm{G} 2$ is the most suitable phase for irradiation, because exposure of G0 cells to the adaptive dose stimulates reparative mechanisms in the cell [5]. Over $48 \mathrm{~h}$, the cells complete the mitotic cycle and the damaging dose is applied during G2 phase of the next cell cycle. This scheme allows evaluation of the response of adapted cells to the damaging dose applied after the cell passes the main checkpoints (pre- and postreplicative reparations).

The level of $\gamma$-interferon was measured by ELISA using commercial Bender MedSystems kits (Austria) according to manufacturer's instruction.

\section{Results}

The radioadaptive response was observed in 7 individuals of the control group (78\%); the mean age in the control group is 23 years. In the group with total flight time $<1000 \mathrm{~h}$ (mean age 33 years), the adaptive response was noted in 10 individuals $(59 \%)$. In the group with total flight time $>1000 \mathrm{~h}$ (mean age 39 years), the adaptive response was noted in 4 individuals $(33 \%)$. The examined individuals were divided into 2 groups depending on the presence of RAR, and IFN- $\gamma$ production after radiation was measured. It was shown that at doses 0.05 Gy or 0.5 Gy no differences between groups were detected. Exposure with these doses sequentially in $48 \mathrm{~h}$ interval resulted to differently directed changes: lymphocytes of individuals with RAR produced more IFN- $\gamma$ than before while cells of persons without RAR made it less.

As is seen, the groups differed by age. It is known that the incidence of spontaneous chromosome aberrations is a conservative parameter; it does not depend on gender, but depends on age. The number of aberrant cells in healthy individuals varies from 0 to $5 \%$, being $\sim 1 \%$ at the age of 20 40 years and increasing to $1.5 \%$ to $40-50$ years. [6]. The examined groups of pilots fall into the same age group, hence, the age does not play a role in elevation of the percentage of aberrant cell.

Similar analysis of the incidence of persons with RAR in groups with different flight time showed that the percent of individuals with RAR decreases with increasing the flight time (Fig.3).

The ratio of aberration observed after exposure to the damaging dose to the same parameter after exposure to $\mathrm{AD}+\mathrm{CD}$ is usually considered as a qualitative sign, but analysis showed that the most part of the sample by RAR coefficient fell into the $2 \sigma$ range. Only one person in the group with flight time $<1000 \mathrm{~h}$ and one person in the group with flight time $>1000 \mathrm{~h}$ fell beyond this interval (RAR coefficients 2.4 and 2.5 , respectively). This probably suggests that these individuals are characterized by increased radiation resistance.

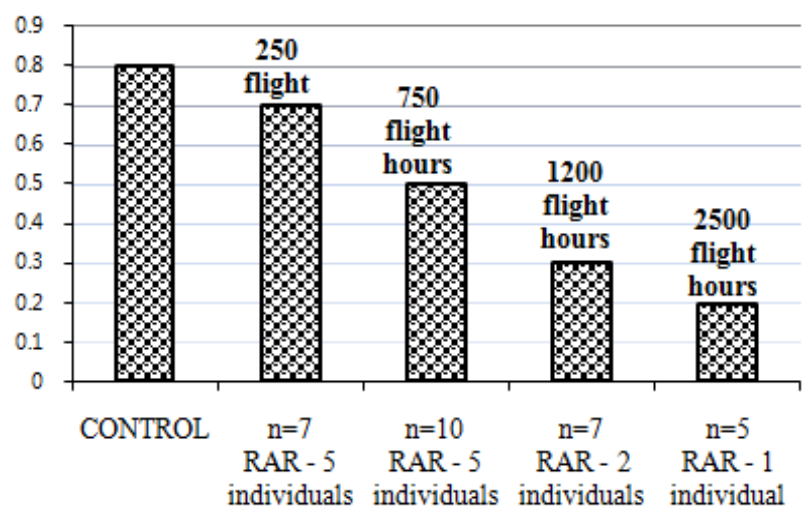

Figure 3. Incidence of individuals with RAR depending on flight time

A correlation was revealed between the total flight time and RAR coefficients $(\mathrm{r}=-0.37, \mathrm{p}=0.02)$ : RAR coefficients decreased with increasing the total flight time.

On the whole, the data presented here suggest that the quality of nonspecific adaptive mechanisms in the groups decreases with increasing the total flight time. Chronic exposure to genotoxic factors in doses surpassing the background level impairs reparative processes and determines the absence of AR.

The immune and humoral systems play an important role in AR formation at the organism level [7]. The magnitude of AR can be related to the relative content of blood cells, in particular, lymphocytes.

Since we examined healthy individuals, no pronounced differences in blood parameters were expected. However, certain differences in the lymphocyte and monocyte counts were revealed. Comparison of these values with published data on normal content of these cells in the blood of healthy individuals revealed a slight increase in these parameters in the studied cohort. It is also worthy of note that lymphocyte 
count was higher in individuals with AR than in subjects without it. The total number of lymphocyte decreased with increasing the flight time. Opposite changes were observed for monocyte count: it increased with increasing the flight time and in the absence of AR (Fig. 4).

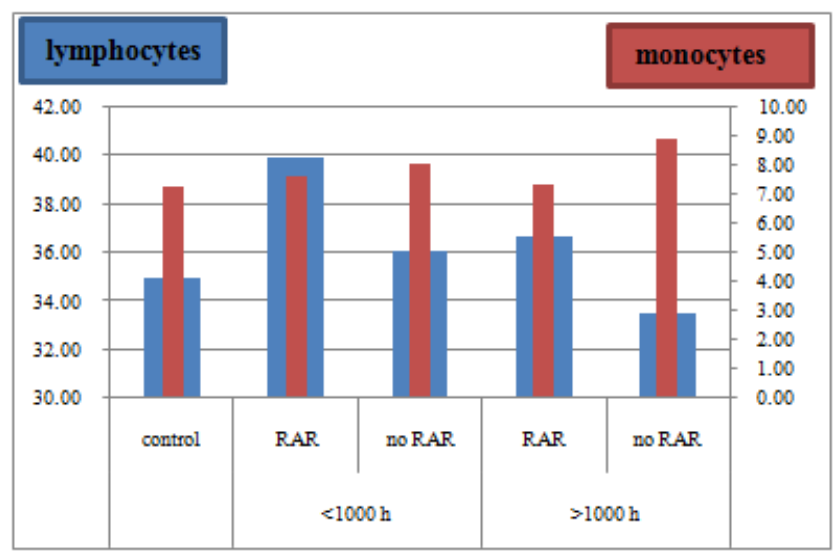

Figure 4. The number of mononuclear cells in the blood of pilots.

These changes in blood cells can result from adaptation to flight factors, which at the molecular level is the cause of AR presence or absence.

One of the cytokines produced in response to CpG-DNA is interferon. It produces a direct inhibitory effect on replication in viral infection and demonstrates pleiotropic immunomodulatory activity; in particular, it potentiates cytotoxicity of natural killers and macrophages, activates and maintains $\mathrm{T}$ cells, induces production of $\gamma$-interferon by human $\mathrm{CD}^{+} \mathrm{T}$ cells [8], and increases cytotoxicity of $\mathrm{T}$ cells via production of TNF triggering apoptosis. It acts via a receptor complex consisting of a ligand-binding chain and a regulatory site. Binding of $\gamma$-interferon is followed by receptor dimerization. Then, receptor-associated kinases Jak1 and Jak2 transphosphorylate each other, which leads to their activation. Phosphorylation of the cytoplasmic domain of the receptor mobilizes STAT1 protein transcription activator that passes through the nuclear pores in the form of a dimer and activates genes responsible for the production of $\gamma$-interferon via binding with the corresponding sequence [9]. Then, STAT1 is dephosphorylated and released into the cytoplasm.

The process of interferon production is influenced by various factors, e.g. UV radiation inhibits phosphorylation of STAT1, thus attenuating the biological effects of $\gamma$-interferon [10].

The list of cytokines mediating the effect of radiation on cells is constantly increased. Recent studies have demonstrated that interleukin-24 exhibits antitumor activity realized by the bystander mechanism [11]. Introduction of mda-7 gene into lung carcinoma cells improves their radioresistance through intensification of apoptosis; however, this effect was not observed in normal fibroblasts [12]. Authors have demonstrated that radiation activated c-jun Nterminal MAP kinase (JNK); at the same time, usual elements p53 and Fas were not involved into the improvement of radioresistance of tumor cells. Synergistic action of the studied factors or transfected gene and radiation-stimulated free-radical processes in the downregulation of the expression of anti-apoptotic protein BCL$\mathrm{X}_{\mathrm{L}}$ and activation of $\mathrm{BAX}$ protein expression resulting in increased radiosensitivity in vitro has been demonstrated. Enhanced expression of anti-apoptotic protein BCL- $\mathrm{X}_{\mathrm{L}}$ protected RT2 rat glioma cells from growth inhibition and impairment of survival caused by combined effects of mda-7 and ionizing radiation [13].

It is known that interferons can produce a clastogenic effect. In particular, experiments performed by G. P. Makedonov et al. have shown that human lymphocytes treated with lymphoblast interferon were less sensitive to subsequent $\gamma$-irradiation in a dose of 2 Gy [14]. The authors attributed this effect to stimulation of excision and postreplicative reparation and induction of DNA double break repair under the effect of interferon.

$\gamma$-Interferon, in turn, produces an irreversible cytotoxic effect on transformed cells. Thus, the amount of $\gamma$-interferon allows evaluation of cell death. The content of $\gamma$-interferon in the culture medium was measured by ELISA ("Bender MedSystems", Austria). PHA used in the experiments is a potent inductor of $\gamma$-interferon; this fact that can explain insignificant changes after exposure to adaptive and damaging radiation doses. At the same time, the cumulative dose $(0.05+0.5 \mathrm{~Gy})$ induced opposite changes in the production of $\gamma$-interferon in individuals demonstrating ($7.92+5.42 \%)$ or not demonstrating $(14.82+16.67 \%)$ the adaptive response (Fig. 5).

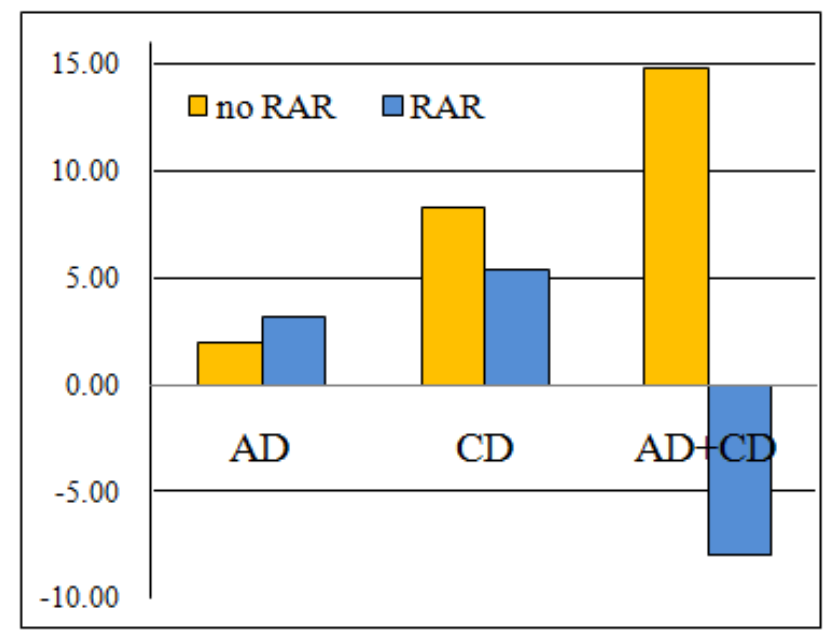

Figure 5. The ratio of interferon concentrations in samples of fluid supernatant after culturing cells in RAR. On the ordinate axis - the percentage of excess compared to the control

Since repair mechanisms are not sufficiently activated in donors without adaptive response, the increase in the interferon level can be a humoral regulation at the cellular level, which is stimulated by the remaining level of chromosome aberrations at $0.5 \mathrm{~Gy}$.

Small sample size does not allow definitive conclusions about the dependence of the immune response on individual sensitivity to radiation exposure; however, revealed trends 
seem to be very promising for further research.

The monotonic decrease in the frequency of adaptive response in pilots and a correlation between AR coefficients with the number of flight hours suggest that flight factors cause gradual exhaustion of the adaptive resource in the majority of examined individuals. At the same time, some individuals with long flight hours demonstrated pronounced AR. The overrange in these individuals (AR beyond $2 \sigma$ ) can be a manifestation of increased individual resistance to flight factors.

The role of cytokines in the development of various processes is often deduced from their plasma or serum levels. This approach has many advantages, for instance, the possibility of rapid isolation and processing of a large number of samples. However, measurement of the concentration reflects systemic effects of cytokines, including their interaction with each other and with hormones and other substances as well as production by immune and nonimmune cells (epithelial cells and fibroblasts), though these aspects are not often taken into account. Thus, this approach has some limitations. The serum level of cytokines is influenced by receptor binding, thermal degradation, and urinary excretion. Since the cytokines are secreted by the paracrine mechanism and their main function is mediation of cell-cell interaction, measurement of cytokine concentration in blood serum can only indirectly reflect realization of this function [15].

High prognostic value of measuring the concentration of cytokines and products of their activity (neopterin, $\beta_{2^{-}}$ microglobulin, specific soluble receptors) in some pathologies is beyond doubts. However, the absolute concentrations of cytokines and other markers of immune system activation measured in different laboratories can differ by two orders of magnitude [16]. It should be noted that these differences were detected when processing both the sera and supernatants; hence, they were determined by the quality of the used kits.

In our experiments, supernatants were used and the changes were oppositely directed, which, together with the medium absolute values (according to published data), allows concluding the correctness of the obtained data.

\section{References}

[1] Cavallo D., Ursini C.L., Carelli G., Iavicoli I. et. al., "Occupational exposure in airport personnel: Characterization and evaluation of genotoxic and oxidative effects," Toxicology, Apr., 2006, pp. $16-18$.

[2] Radiation Exposure and High-Altitude Flight, NCRP Commentary, 12, 1995.

[3] Bochkov N.P., Popova N.A., Katosova L.D. and al., "Extraordinarily high level of chromosomal variability in culture human peripheral blood lymphocytes,"Genetika, vol. 35, No. 6, 1999, pp. 838-841. (in Russian)
[4] Antoschina M.M., Fesenco E.V., Nasonova V.A., Ryabchenco N.I., "Adaptive response after preliminary irradiations of human lymphocytes," $27^{\text {th }}$ Annual Meeting of the European Society for Radiation Biology, vol. 32,1997, pp.407-408.

[5] Mortazavi S.M.J., Mozdarani H. "The search for a possible optimum adapting dose under the optimum irradiation time scheme in cultured human lymphocytes,'International Journal of Low Radiation, vol. 3, No. 1,2006, pp.74-82.

[6] B.S. Fedorenko, Iu.I. Voronkov, G.P. Snigireva, V.A. Shevchenko, S.V. Druzhinin, Iu.A. Akatov, V.V. Tsetlin, "Effect of space flight factors on health of cosmonauts in the near and late term after space flights,"Radiats Biol Radioecol, Nov-Dec, vol. 42, No. 6, 2002, pp.765-8. (in Russian)

[7] Nenoi M, Wang B, Vares G., "In vivo radioadaptive response: A review of studies relevant to radiation-induced cancer risk," Hum Exp Toxicol., 2014 Jun 12, pii: 0960327114537537. [Epub ahead of print]

[8] David J.T.Jr., J.R. Patterson, C.Velasco-Gonzalez, E.N. Carroll, J. Trinh, D. Edwards, A.Aiyar, B. Finkel-Jimenez, and A.H. Zea, "Interferon-Gamma-Induced Nitric Oxide Inhibits the Proliferation of Murine Renal Cell Carcinoma Cells," International Journal of Biological Sciences, vol. 8, No. 8,2012, pp. 1109-1120. doi: 10.7150/ijbs.4694

[9] Kaffman A., O'Shea E.K., "Regulation of nuclear localization: a key to a door," Annu. Rev. Cell Dev. Biol, vol.15, 1999, pp.291-339.

[10] Lillemeier B.F., Koster M., Kerr I.M., "STAT1 from the cell membrane to the DNA,"EMBO J, vol. 20, No. 10, 2001, pp.2508-2517.

[11] Gupta P., Su Z., Lebedeva I.V. et al., "mda-7/IL-24: Multifunctional cancer-specific apoptosis-inducing cytokine," Pharmacol. Therap., vol.11, No. 3, 2006, pp.596-628.

[12] Kawabe S., Nishikawa T., Munshi A., "Adenovirus-mediated mda-7 gene expression radiosensitizes non-small lung cancer cells via TP53-independent mechanisms," Molec. Ther., vol. 6, 2002, pp.637-644.

[13] Yacoub A., Mitchell C., Lister A. et al., "Melanoma differentiation-associated 7 (interleukin 24) inhibits growth and enhances radiosensitivity of glioma cells in vitro and in vivo," Clin. CancerRes., vol.9, 2003, pp.3272 - 3281.

[14] Makedonov GP, Tskhovrebova LV, Vasil'eva IM, Zasukhina GD.,"Radioadaptive response and antimutagenic effect of interferon have common pathways of cell protection against gamma radiation,'Dokl Akad Nauk, vol. 359, No. 6, 1998, pp. 838-40.(in Russian)

[15] Jason J., Archibald L.K., Nwanyanwu O.C. et al., "Comparison of serum and cell-specific cytokines in humans,"Clin. Diag. Lab. Immunol., vol.8, No.6, 2001, pp.1097- 1103.

[16] Fahey J.L., Aziz N., Spritzler J. et al., "Need for external proficiency testing program for cytokines, chemokines, and plasma markers of immune activation," Clin. Diag. Lab. Immunol., vol.7, No.4, 2000, pp.540-548. 